\title{
Cellular immune response of the Asian corn borer, Ostrinia furnacalis (Lepidoptera: Pyralidae), to infection by the entomopathogenic fungus, Beauveria bassiana
}

\author{
Dongxu SHEN ${ }^{1, *}$, Miao LI ${ }^{1, *}$, Yuan CHU ${ }^{1}$, Minglin LANG ${ }^{2}$ and Chunju AN ${ }^{1, * *}$ \\ ${ }^{1}$ Department of Entomology, China Agricultural University, 100193, Beijing, China; e-mails: shendongxu0311@163.com; \\ limiao@ksu.edu; chuyuan0617@sina.com; anchunju@cau.edu.cn \\ ${ }^{2}$ College of Life Science, University of Chinese Academy of Sciences, 100049, Baoding, China; \\ e-mail: langminglin@tsinghua.org.cn
}

Key words. Lepidoptera, Pyralidae, Ostrinia furnacalis, cellular immune response, haemocyte, phagocytosis, nodulation, Beauveria bassiana, entomopathogenic fungus

\begin{abstract}
The term cellular immune response refers to haemocyte-mediated responses, including phagocytosis, nodulation, and encapsulation. In the present study, we identified five types of circulating haemocytes in larvae of the haemolymph of the Asian corn borer, Ostrinia furnacalis (Guenée), including granulocytes, oenocytoids, plasmatocytes, prohaemocytes, and spherulocytes. The relative number of total free haemocytes per larva decreased significantly $0.5,24$, and $36 \mathrm{~h}$ after the injection of Beauveria bassiana conidia. Upon conidia challenge, both phagocytosis and nodulation were observed in the collected haemolymph from $O$. furnacalis larvae. In addition, plasma was found to be necessary for both phagocytosis and nodulation. Therefore, we here confirm that phagocytosis and nodulation are involved in $O$. funacalis larvae during their fight against infection by $B$. bassiana, and further, that the cellular immune response of $O$. furnacalis helps eliminate the invading organisms despite the fact that not all the fungal conidia are killed.
\end{abstract}

\section{INTRODUCTION}

Insects lack acquired immunity and rely mainly on the well-developed innate immune system to defend against infection by pathogens or parasites (Cherry \& Silverman, 2006; Eleftherianos et al., 2010; Jiang et al., 2010; Castillo et al., 2011), comprising both humoral and cellular defense responses (Cherry \& Silverman, 2006; Lemaitre \& Hoffmann, 2007). The humoral immune response includes antimicrobial peptide synthesis, melanization and coagulation of haemolymph, and the production of reactive intermediates of oxygen and nitrogen (Nappi \& Ottaviani, 2000; Cerenius \& Soderhall, 2004; Kanost et al., 2004; Mastore et al., 2015b). The term cellular immune response refers to haemocyte-mediated responses, including phagocytosis, nodulation and encapsulation (Lavine \& Strand, 2002; Jiravanichpaisal et al., 2006; Mastore et al., 2015a). Upon infection, cellular and humoral immune responses are initiated simultaneously to efficiently eliminate pathogenic microorganisms. However, despite the present relatively comprehensive understanding of the humoral immune re- sponse of insects, much less is known about the cellular immune response of these animals.

The majority of studies on insect cellular immune responses have been conducted on dipteran and lepidopteran insects such as flies, mosquitoes, and hornworms (Jiravanichpaisal et al., 2006; Ferrandon et al., 2007; Hillyer \& Strand, 2014; Honti et al., 2014; Hillyer, 2016, and references therein). Circulating haemocytes (insect blood cells) play critical roles in insect cellular immune responses. There are at least five types of haemocytes commonly found in insects: granulocytes (= granular cells), oenocytoids, plasmatocytes, prohaemocytes, and spherulocytes (= spherule cells) (Browne et al., 2013). In lepidopteran larvae, granulocytes and plasmatocytes together usually comprise $>50 \%$ of the total haemocytes (Lackie, 1983; Strand \& Pech, 1995). The number of circulating haemocytes obviously varies during the cellular immune response. For example, the relative number of circulating haemocytes in Metarhizium anisopliae-challenged Eastern subterranean termites, Reticulitermes flavipes (Kollar)

\footnotetext{
* Both authors contributed equally to this work.

** Corresponding author; e-mail: anchunju@cau.edu.cn
} 
(Blattodea: Isoptera: Rhinotermitidae) or Phytomonas serpens-induced Large milkweed bugs, Oncopeltus fasciatus (Dallas) (Hemiptera: Lygaeidae) increased significantly, while the pool of circulating haemocytes became depleted in Bacillus thuringiensis-infected Greater wax moths, Galleria mellonella (L.) (Lepidoptera: Pyralidae) (Chouvenc et al., 2009; Alves e Silva et al., 2013). In Macrocentrus cingulum-parasitized Asian corn borer moth larvae, Ostrinia furnacalis (Guenée) (Lepidoptera: Crambidae), the total number of haemocytes remained unchanged for 4 days, but decreased significantly 5 days post parasitization (Hu et al., 2003).

Haemocytes mainly function through various processes including phagocytosis, nodulation and encapsulation to entrap and clear invading pathogens from the haemolymph (Lavine \& Strand, 2002; Jiravanichpaisal et al., 2006). They also respond to external injury by participating in clot formation (Theopold et al., 2004; Scherfer et al., 2006). Phagocytosis refers to the engulfment of biotic or small abiotic targets by an individual cell (Yokoo et al., 1995; Hernandez et al., 1999; Borges et al., 2008; Brivio et al., 2010). Nodulation is a complex multi-step process in which multiple haemocytes aggregate and entrap a large number of microorganisms, while encapsulation refers to the binding of multi-cellular haemocytic aggregates to larger targets like parasitoids, nematodes and chromatography beads (Ratcliffe \& Gagen, 1977; Jiravanichpaisal et al., 2006). Unlike phagocytosis, nodulation and encapsulation result in the formation of a multilayered, overlapping sheaths of haemocytes around the invader. In Lepidoptera, only granulocytes and plasmatocytes are reported to be involved in these three processes (Browne et al., 2013).

The Asian corn borer, $O$. furnacalis is a serious agricultural pest on several crops, especially corn. It is perceived as being the worst pest on corn in the western Pacific region of Asia (Afidchao et al., 2013). Control of this pest with chemical insecticides is currently hampered by the cryptic nature of larval behaviour. Furthermore, excessive use of chemical insecticides has also led to severe environmental problems and to the evolution of insecticide resistance in these moths (Naqqash et al., 2016; Taskin et al., 2016). The potential for suppression of Asian corn borer larvae by the entomopathogenic fungus, Beauveria bassiana (Bals.-Criv.) Vuill. (1912) (Hypocreales: Clavicipitaceae) made the utilization of this fungus a promising alternative control agent of the pest (Wagner \& Lewis, 2000). However, the complex interactions between $O$. furnacalis and B. bassiana are still largely unknown. It is, for example, still not known how Asian corn borer responds to the infection of B. bassiana with respect to cellular immunity.In the present study, we identified and described five types of haemocytes in the haemolymph of $O$. furnacalis, including granulocytes, oenocytoids, plasmatocytes, prohaemocytes, and spherulocytes. We also counted the numbers of free haemocytes with or without the injection of $B$. bassiana conidia. Lastly, we observed the involvement of phagocytosis and nodulation in $O$. funacalis following challenge by B. bassiana.

\section{MATERIAL AND METHODS}

\section{Insect rearing}

Asian corn borer larvae were reared on an artificial diet at $28^{\circ} \mathrm{C}$ at a relative humidity of $70-90 \%$ and photoperiod of $16 \mathrm{~L}: 8 \mathrm{D}$ (Liu et al., 2014).

\section{$B$. bassiana culture and conidia suspension preparation}

B. bassiana strain Bb-252-GFP which had been modified and labeled with green fluorescent protein (GFP) was kindly provided by Dr. Weiguo Fang from Zhejiang University. It was cultured on potato dextrose agar plates at $25^{\circ} \mathrm{C}$ and at $80 \%$ humidity. Conidia used for $O$. furnacalis infection were harvested from 3-4 week old cultures by scraping the surface of the mycelia into sterile deionized water containing $0.1 \%$ Tween- 80 . Conidia were separated from other mycelial structures over a sterile funnel packed with autoclaved glass wool, washed two times with $\mathrm{ddH}_{2} \mathrm{O}$ by centrifugation at 4,000 rpm, counted and diluted to $2 \times 10^{5}$ conidia $/ \mu 1$. Freshly prepared conidia were used for all experiments.

\section{Collection of haemolymph and determination of haemocyte types}

Day zero fifth instar $O$. furnacalis larvae were rinsed with $75 \%$ alcohol, then haemolymph was collected by cutting the abdominal proleg with scissors. To determine the haemocyte type, $5 \mu \mathrm{l}$ of sample was uniformly daubed on slides and fixed with formaldehyde for $20 \mathrm{~min}$ at room temperature. Nuclei of cells were stained with DAPI (4',6-diamidino-2-phenylindole) for $10 \mathrm{~min}$, and cell membrane was stained with phalloidin for $1 \mathrm{~h}$. After washing three times with sterile phosphate-buffered saline (PBS), images of haemocytes were taken under a fluorescence microscope. In addition, Giemsa was used to stain haemocytes as described previously (Sanghamitra et al., 2014). After fixing with formaldehyde, haemocytes were stained with Giemsa for $30 \mathrm{~min}$ and observed by light microscopy.

\section{Free circulating haemocyte count}

Day zero fifth instar larvae were injected into the haemocoel with $3 \mu \mathrm{l} \mathrm{B}$. bassiana $\left(1 \times 10^{4}\right.$ conidia/ $\left.\mu \mathrm{l}\right)$ or sterile PBS (as a control) using a micro-injector. The haemolymph was collected at $0.5,1,12,24,36$, and $48 \mathrm{~h}$ post infection (hpi), as described above. A $5 \mu \mathrm{l}$ of fresh haemolymph per larva was diluted 10 -fold with ice cold $1 \times$ PBS buffer. Total haemocyte counts were immediately performed using a haemocytometer and were expressed as the number of haemocytes per ml of undiluted haemolymph. One larva was used for each sample. One sample was calculated twice. Ten larvae were sampled at each time point.

\section{In vivo phagocytosis and nodulation with haemocytes challenged by $B$. bassiana}

For fungus-challenging experiments, day zero fifth-instar $O$. furnacalis larvae were injected with $3 \mu \mathrm{l}$ of diluted $B$. bassiana conidia suspension $\left(1 \times 10^{5}\right.$ conidia/ $\left.\mu \mathrm{l}\right)$. After $2 \mathrm{~h}, 5 \mu \mathrm{l}$ of haemolymph containing $B$. bassiana conidia was collected and uniformly daubed on slides. The following fixation and staining of haemocytes were the same as described above. The stained haemocytes were then observed for phagocytosis and nodulation under a fluorescence microscope.

\section{Effect of plasma on phagocytosis and nodulation in O. furnacalis}

In order to investigate the effects of plasma (haemocyte-free haemolymph) alone on phagocytosis and nodulation in vitro, 50 $\mu 1$ of haemolymph collected from fifth instar larvae was centrifuged at $300 \times \mathrm{g}$ for $2 \mathrm{~min}$ at $4^{\circ} \mathrm{C}$. The haemocytes thus obtained were washed 3 times with cold $1 \times$ PBS (each for $5 \mathrm{~min}$ ) and re-suspended in $50 \mu \mathrm{l}$ of SF9 cell culture medium. Then $3 \mu \mathrm{l}$ of 

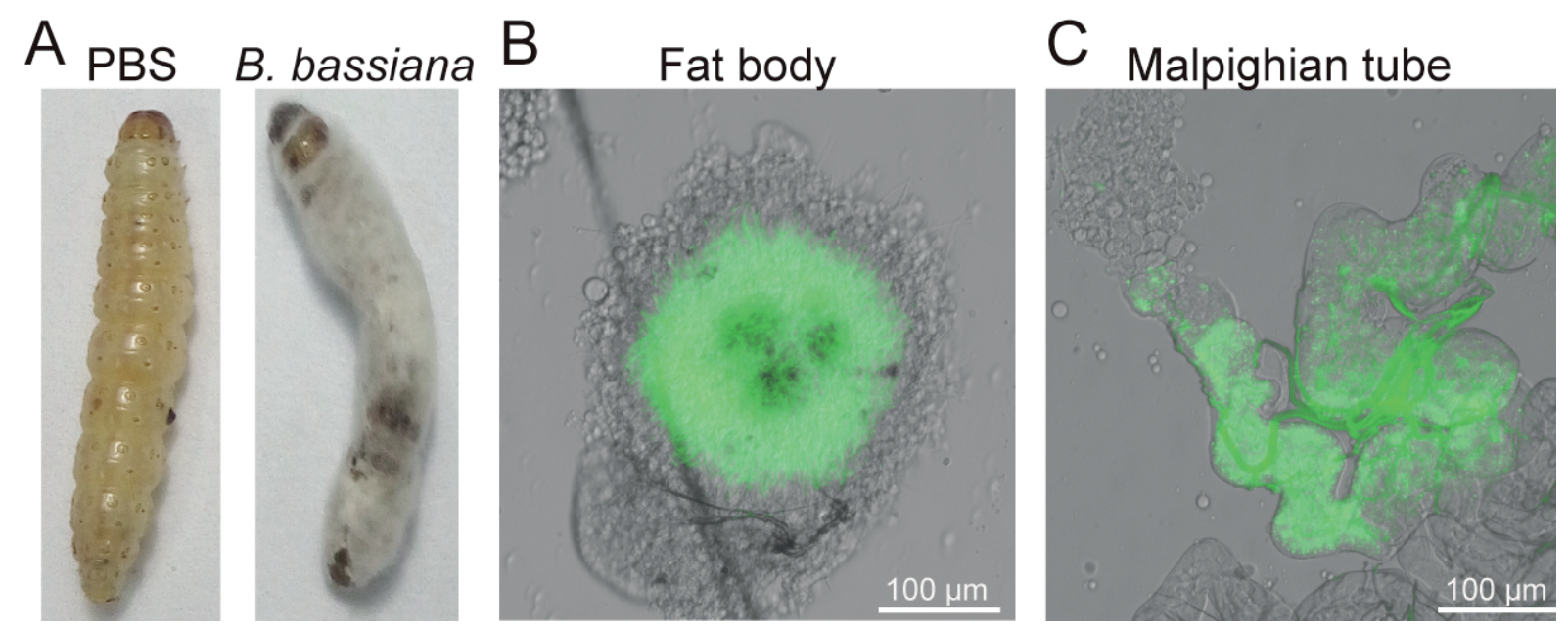

Fig. 1. Observation of infection of $O$. furnacalis larvae by $B$. bassiana conidia. Each larva was injected with $1 \times 10^{5} B$. bassiana conidia (sterile PBS was used as a control) and observed continuously until its death. A - images of $O$. furnacalis larvae (control and infected) taken 3 days after injection. B. bassiana-injected larva was covered by white hyphae. B and $\mathrm{C}-$ fat body and Malpighian tubule from $B$. bassianainfected larva. Tissues were dissected $18 \mathrm{hpi}$ and viewed under a fluorescence microscope. GFP-labeled conidia are shown in green.

B. bassiana conidia suspension $\left(1 \times 10^{5}\right.$ conidia/ $\left.\mu \mathrm{l}\right)$ was added to the medium. After incubation for $2 \mathrm{~h}$ in vitro, nodulation and phagocytosis of $B$. bassiana conidia were observed by fluorescence microscopy.

\section{RESULTS}

\section{Germination and proliferation of $B$. bassiana} conidia in $O$. furnacalis larvae

In our experimental conditions, none of the Asian corn borer larvae injected with a control solution showed any sign of sickness, and all survived to pupation. No B. bassiana conidia were found either on the surface or in the internal tissues or the haemocoel in the control larvae. However, of ten $B$. bassiana conidia-injected larvae, eight died at 3 days after challenge and the remaining two died at 4 and 5 days after infection, respectively. After leaving these B. bassiana-killed larvae to decompose for 2 days, the cadavers were covered by white B. bassiana hyphae (Fig.
1A). In fact, the conidia germination and hyphae growth were observed in the fat bodies and Malpighian tubules in infected larvae starting from $18 \mathrm{hr}$ after injection (Fig. 1B and $1 \mathrm{C}$ ). Signs of humoral and cellular immune responses were observed during this process (see below), although the immune responses failed to eliminate the invading conidia, which finally occupied the entire larval body. Here, we focus only on the cell-mediated immune responses in Asian corn borer larvae found following attack by the invading entomopathogenic fungus.

\section{Identification of free circulating haemocytes in O. furnacalis larvae}

Considering circulating haemocytes have important roles in the cell-mediated immune responses of insects against parasites or pathogens, we started our work by first identifying the number of free haemocytes in O. furnacalis larvae. Five morphological types of the circulating haemo-
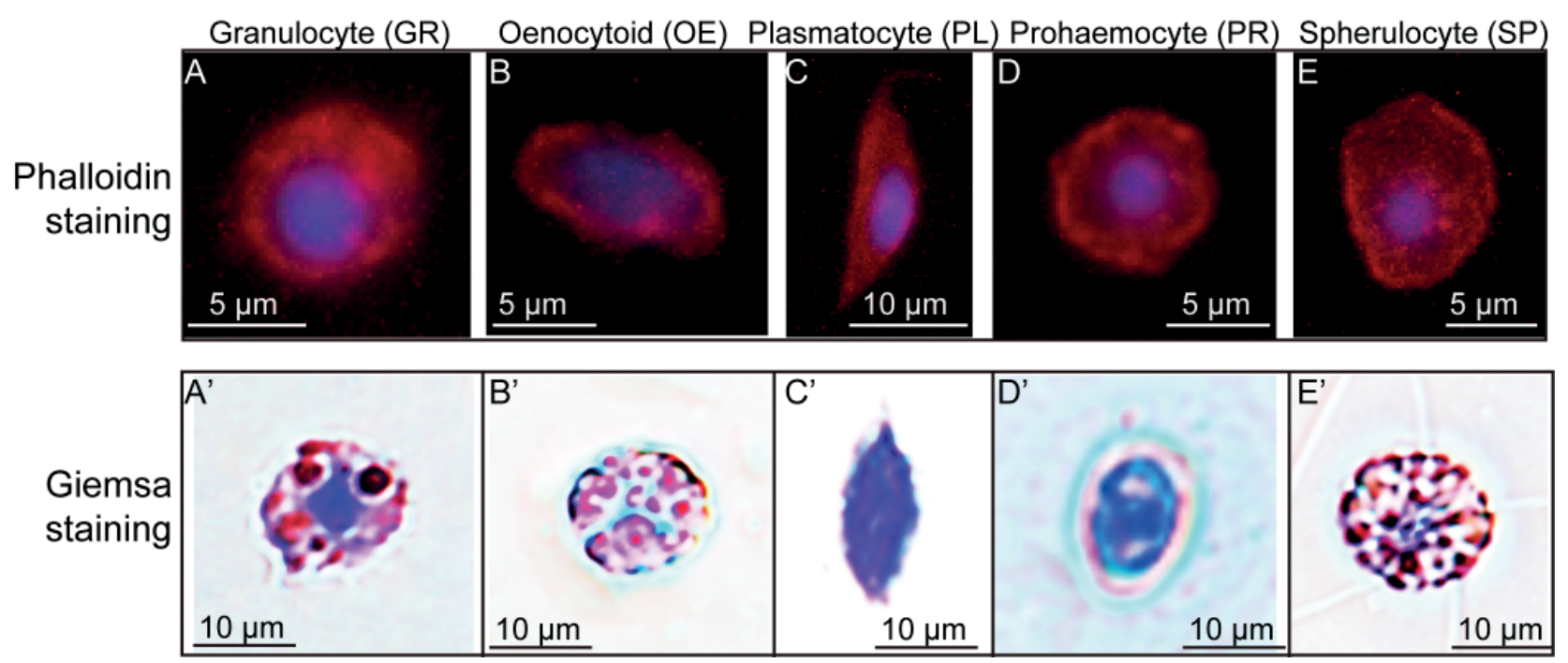

Fig. 2. Images of circulating haemocytes from $O$. furnacalis larvae. Haemocytes were recovered from haemolymph of $O$. furnacalis larvae, stained with DAPI (blue) and phalloidin (red) or Giemsa, and viewed under a fluorescence (upper panel) or light (lower panel) microscope. A, A' - granulocyte; B, B' - oenocytoid; C, C' - plasmatocyte; D, D' - prohaemocyte; E, E' - spherulocyte. 


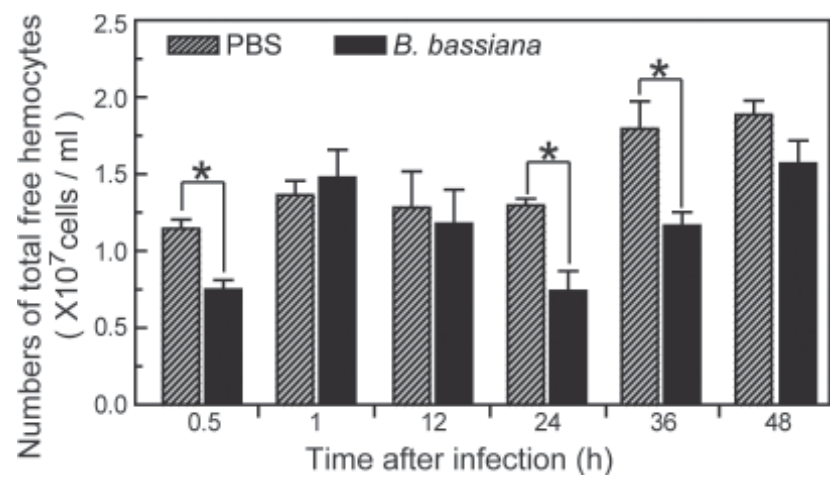

Fig. 3. Total haemocyte count of $O$. furnacalis larvae infected with $B$. bassiana conidia. Each larva was injected with $3 \times 10^{4} \mathrm{~B}$. bassiana conidia (sterile PBS was used as a control) and the cellular density of the haemocytes was determined using a haemocytometer chamber. Each bar represents the mean \pm standard error ( $n$ $=10)$. The asterisk indicates a significant difference from the control (unpaired $t$ test for the values at particular time point, $p<0.05$ ) Lack of asterisk means the difference is not significant $(p>0.05)$.

cytes were characterized in the larval haemolymph, including granulocytes, oenocytoids, plasmatocytes, prohaemocytes, and spherulocytes (Fig. 2). Granulocytes are usually spherical or ovoid, with a 10 to $20 \mu \mathrm{m}$ diameter. They have large nuclei and numerous granules in the cytoplasm. After staining with Giemsa, the nucleus appears blue and purple while the granules appear pink (Fig. 2A, 2A'). Oenocytoids display an irregular shape with dense cytoplasmic granules and inclusions. The nucleus is invisible under light microscopy. So oenocytoids show pink after Giemsa staining (Fig. 2B, 2B'). Plasmatocytes are spindle-like or leaf-like cells with cytoplasmic processes and granules in the cytoplasm. Because of the large nucleus, plasmatocyte appears blue and purple following reaction with Giemsa (Fig. 2C, 2C').
Prohaemocytes are the smallest cells in haemolymph with a spherical shape. The nucleus is large, centrally located, and fills the cell so that the basophilic cytoplasm occupies just a limited layer around the nucleus (Fig. 2D, 2D'). Spherulocytes are highly polymorphic, with a spherical to irregular shape. Numerous small spherical inclusions are observed in the cytoplasma (Fig. 2E, 2E'). Additionally, spherulocytes are very fragile and break easily to release spherules during collection. Prohaemocytes and spherulocytes are rarely observed in the haemolymph. They were not always captured in the visual field in our experiments.

\section{Count of free circulating haemocytes}

As a first step to investigate the roles of haemocytes in the cellular immune response of Asian corn borer, we counted the numbers of total circulating haemocytes at different time points after the infection of $B$. bassiana conidia. As shown in Fig. 3, control O. furnacalis larvae mock challenged with PBS had an average total haemocyte count of $1.50 \times 10^{7}\left( \pm 0.42 \times 10^{7}\right.$ over the experiment $)$. When the larvae were infected by $B$. bassiana conidia, free circulating haemocytes significantly decreased at 0.5 $\mathrm{h}$ after treatment, and thereafter showed no significant differences compared with the control at 1 and $12 \mathrm{hpi}$ (Fig. 3 ). However, the number of free haemocytes significantly decreased again in response to $B$. bassiana infection after 24 and $36 \mathrm{~h}$, and then showed no significant difference at 48 hpi (Fig. 3).

\section{Cellular phagocytosis of $B$. bassiana conidia in O. furnacalis larvae}

When GFP-labeled B. bassiana conidia were injected into Asian corn borer larva haemocoel, the cellular uptake of injected conidia was observed by fluorescence microsco-
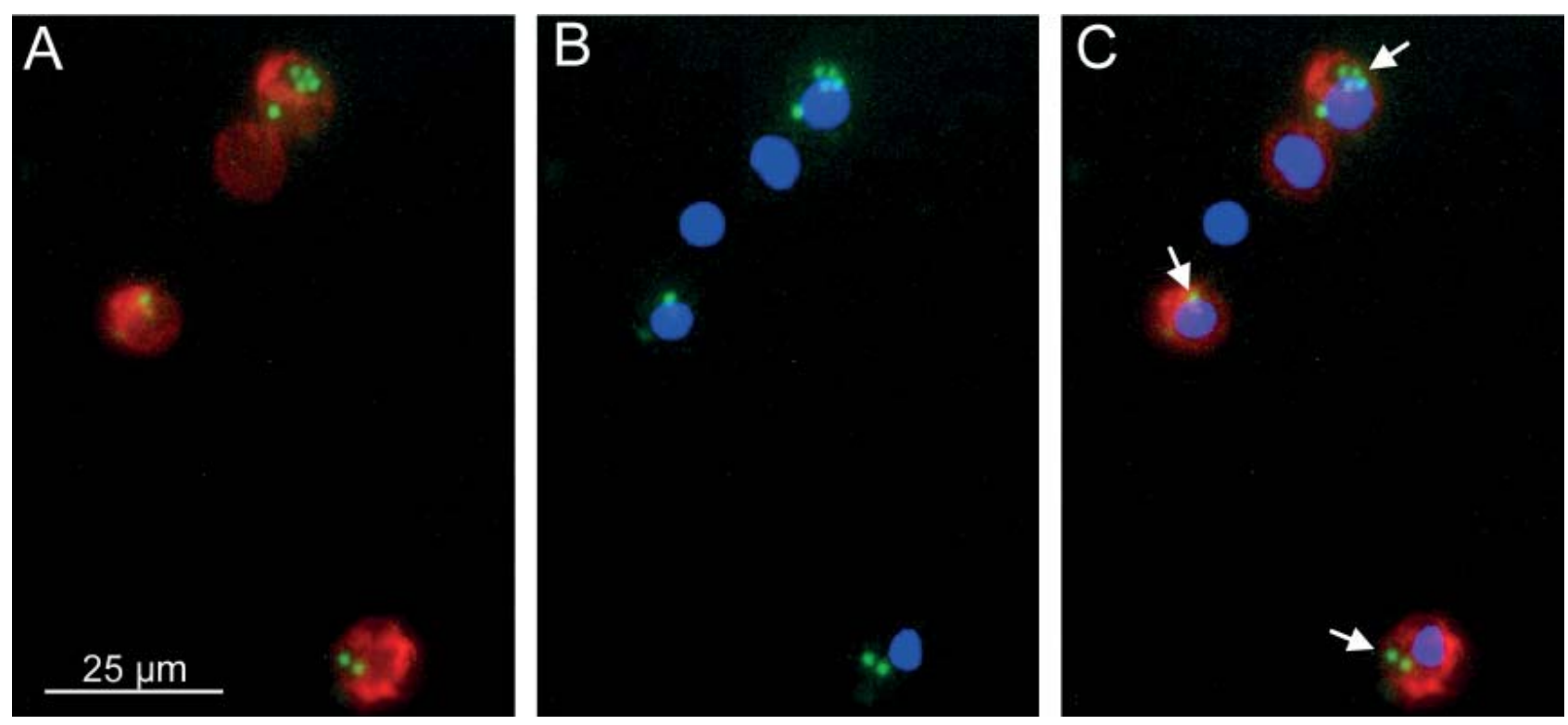

Fig. 4. In vivo phagocytosis of fluorescent $B$. bassiana conidia by $O$. furnacalis haemocytes. $3 \times 10^{5} B$. bassiana conidia were inoculated into $O$. furnacalis larvae. Two hours later, haemolymph containing haemocytes was fixed and stained for observation by fluorescence microscopy. A - merger of fluorescent micrographs showing phalloidin-stained cell membrane (red) and GFP-labeled conidia (green). B - merger of fluorescent micrographs showing DAPI-stained nuclei (blue) and GFP-labeled conidia (green). C - merger of fluorescent micrographs showing phalloidin-stained cell membrane (red), DAPI-stained nuclei (blue), and GFP-labeled conidia (green). The phagocytosed conidia were indicated by arrows. 

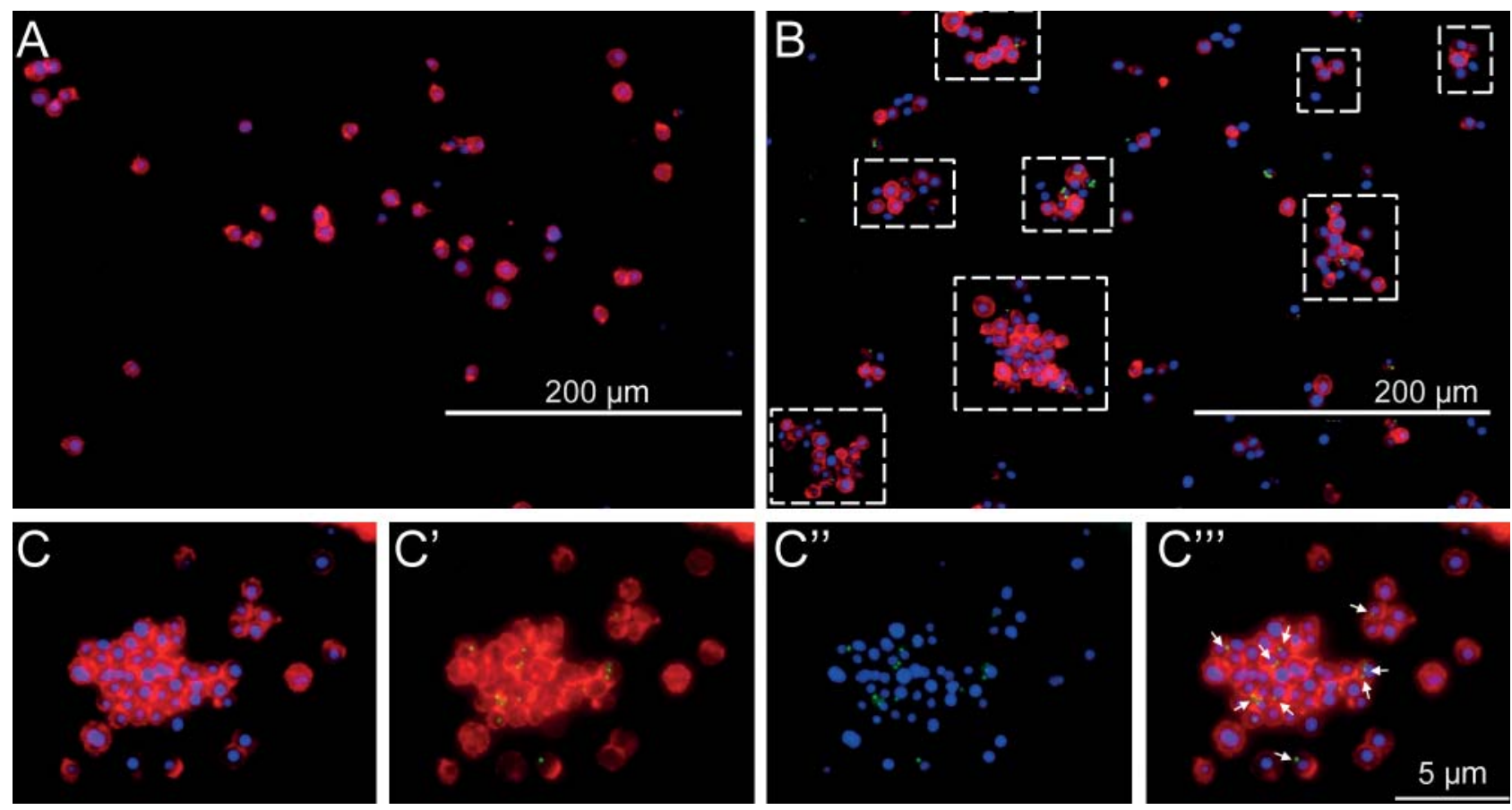

Fig. 5. In vivo nodulation of fluorescent $B$. bassiana conidia by $O$. furnacalis haemocytes. Each day 0 fifth-instar $O$. furnacalis larva was injected with $3 \times 10^{5} \mathrm{~B}$. bassiana conidia or $0.1 \%$ Tween-80 control solution. Two hours later, haemolymph containing haemocytes was fixed and stained for observation by fluorescence microscopy. A - fluorescent image of haemocytes from control larva. Blue: DAPI, red: phalloidin. B - fluorescent image of haemocytes from B. bassiana conidia-injected larva. Nodule formation was marked by boxes. Blue: DAPI, red: phalloidin, green: GFP-labeled conidia. C-C'" - magnified fluorescent images showing the details of nodule formation. C - merger of images showing phalloidin-stained cell membrane (red) and DAPI-stained nuclei (blue); C' - merger of images showing phalloidin-stained cell membrane (red) and GFP-labeled conidia (green); C" - merger of images showing DAPI-stained nuclei (blue) and GFP-labeled conidia (green); C"' - merger of images showing phalloidin-stained cell membrane (red), DAPI-stained nuclei (blue), and GFP-labeled conidia (green). The trapped conidia in a nodule are indicated by arrows.

py. As shown in Fig. 4, haemocytes contained one or multiple phagocytosed conidia. Some haemocytes appeared damaged due to the increased number of intracellular conidia. Here, only granulocytes were taken as an example for cellular phagocytosis in O. furnacalis. Plasmotocytes with phagocytosed GFP-labeled conidia were also detected by fluorescence microscopy (data not shown). However, when haemocytes were isolated from the haemolymph and incubated with GFP-labeled B. bassiana conidia in vitro, no phagocytosed conidium was detected under our test conditions.

\section{Cellular nodulation of $B$. bassiana conidia in 0 . furnacalis larvae}

Besides phagocytosis, nodulation is another important cellular immune response. To determine whether the nodulation response was also involved in O. furnacalis larvae responding to $B$. bassiana conidia, aliquots of haemolymph collected from infected insects were examined by fluorescence microscopy. In the control sample injected with PBS solution, no conidia were seen and the haemocytes were evenly scattered (Fig. 5A). However, in the B. bassiana-inoculated sample, the conidia were trapped in nodular structures formed by haemocytes as early as $2 \mathrm{hpi}$ (Fig. 5B). When one nodule was recorded at higher magnification to examine its finer structural details, the nodule was actually seen to comprise multicellular haemocytic aggregates which formed an overlapping sheath to surround massive conidia (Fig. 5C-5C",). Additionally, we investigated the effect of plasma on $O$. furnacalis nodulation by observing the nodule formation after incubating haemocytes alone and B. bassiana conidia in vitro. No typical nodules were formed in the mixtures containing haemocytes and conidia (data not shown).

\section{DISCUSSION}

Cellular immune responses, including phagocytosis and nodulation, comprise an important part of the innate immune response of insects against infection by invading microorganisms. Studies on the description and molecular mechanism of the cellular immune response have been widely performed in both invertebrates and vertebrates ( $\mathrm{Wu}$ et al., 2015; Laughton et al., 2016). In the present study, we firstly reported how Asian corn borer larvae respond to the infection of B. bassiana with respect to cellular immunity.

We identified five types of haemocytes in Asian corn borer haemolymph: granulocytes, oenocytoids, plasmatocytes, prohaemocytes, and spherulocytes, which are commonly found in other insects (Ling et al., 2005; Browne et al., 2013; Vogelweith et al., 2016). Hu et al. (2003) also identified these five haemocyte types in O. furnacalis larvae under phase contrast microscopy and transmission electron microscopy. Furthermore, granulocytes and plasmatocytes were more common than the other three types of haemocytes, although the exact proportion of granulocytes and plasmatocytes was not recorded due to the rare observation of prohaemocytes and spherulocytes. This was 
consistent with the previous report in which granulocytes and plasmatocytes were described as making up more than $50 \%$ of the circulating haemocytes in lepidopteran larval stages (Strand \& Pech, 1995; Lavine \& Strand, 2002). Hu et al. (2003) even demonstrated that granular haemocytes and plasmatocytes comprised $>90 \%$ of the total haemocyte population. These two types of haemocytes are also the only haemocyte types capable of adhering to foreign surfaces and eliminating the invaders (Elrod-Erickson et al., 2000). They may play important roles in the process of cellular immunity involving recognition and adherence to pathogens.

The density of haemocytes in the haemolymph varies during the life of the insect and also in response to the challenge of pathogens (Bergin et al., 2003; Browne et al., 2013). In this study, the number of total free haemocytes per larva decreased significantly $0.5,24$, and $36 \mathrm{~h}$ after the injection of B. bassiana conidia (Fig. 3). This decrease of free circulating haemocytes suggests a role of haemocytes in the cellular immune response in $O$. furnacalis larvae against B. bassiana conidia. Upon the inoculation of B. bassiana conidia, haemocytes were recruited for cell-mediated immune reactions such as nodulation, which resulted in the depletion of free circulating haemocytes in the haemolymph. Total haemocyte count losses have also been reported following treatment of Reticulitermes flavipes with Metarhizium anisopliae, or the shield bug, Eurygaster integriceps Puton (Hemiptera: Scutelleridae) with $B$. bassiana and its secondary metabolites (Chouvenc et al., 2009; Zibaee et al., 2011). However, the challenge of Oncopeltus fasciatus with Phytomonas serpens induced a significant increase in haemocyte count (Alves e Silva et al., 2013). The observed increase in haemocyte number could be due to pathogen stimulation of the host haematopoiesis, followed by a depletion caused by the immune response activity of the haemocytes.

Phagocytosis is a widely conserved cellular response that occurs in many protozoa and all metazoan phyla. It is initiated by recognition and binding of a target particle to the phagocytic haemocyte (Browne et al., 2013). Molecules responsible for recognition processes, also called pattern-recognition proteins (PRR), are soluble factors freely circulating in the haemolymph, or bound to the haemocyte membrane (Jiravanichpaisal et al., 2006; Browne et al., 2013). During the phagocytosis reaction, individual haemocytes phagocytose either biotic targets such as bacteria, fungi, yeast, and apoptotic cells, or abiotic targets like synthetic beads or India ink particles (Yokoo et al., 1995; Hernandez et al., 1999; Silva et al., 2000). We observed cellular uptake of injected $B$. bassiana conidia in collected haemolymph from challenged Asian corn borer larvae. This suggests that the haemocytes actively take part in the immune response of $O$. furnacalis during $B$. bassiana challenge. On the other hand, no phagocytosed conidium was detected when the isolated haemocytes were incubated with GFP-labeled B. bassiana conidia in vitro. The possible reason was that the molecules responsible for recognition in $O$. furnacalis phagocytosis were in the plasma, or the receptors on the surface of haemocyte alone were not sufficient to initiate the phagocytosis reaction. The phagocytic receptors have been identified in some insects, including Croquemort, dSR-CI, and PGRP-LC in the fruit fly, Drosophila melanogaster Meigen (Diptera: Drosophilidae) (Pearson et al., 1995; Franc et al., 1999; Ramet et al., 2002), BINT2 in the mosquito, Anopheles gambiae Giles (Diptera: Culicidae) (Moita et al., 2006), and integrin in the tobacco hornworm moth, Manduca sexta (L.) (Lepidoptera: Sphingidae) (Zhuang et al., 2008). So far, only integrin $\beta 1$ was revealed to modulate haemocyte spreading and encapsulation in O. furnacalis ( $\mathrm{Hu}$ et al., 2010; Xu et al., 2012). None is characterized as a defined receptor for $O$. furnacalis phagocytosis. Our future work will aim to reveal the phagocytosis mechanism in $O$. funacalis, including the identification of phagocytic receptors.

As another typical cellular immune response, nodulation occurs when multiple haemocytes bind to a cluster of pathogens. Nodule formation is initiated with the microaggregation of haemocytes, which then leads to the entrapment of large numbers of microorganisms in an extracellular material and ends with the melanization of mature nodules (Lavine \& Strand, 2002; Franssens et al., 2006; Zibaee et al., 2011). After Asian corn borer larvae were infected by $B$. bassiana conidia, nodular structures containing conidia were observed in collected haemolymph (Fig. 5). However, isolated haemocytes alone were unable to trap the conidia under our experimental conditions. The possible reason might be formation of a nodule requires that circulating haemocytes change from non-adhesive to adhesive cells that are able to bind to the target and one another and this change requires the involvement of plasma (Clark et al., 1997; Choi et al., 2002; Nardi et al., 2005; Shu et al., 2016). Some molecules have been reported to be involved in this process, such as extracellular matrix (ECM) proteins lacunin and the ligand for peanut agglutinin (PNA) lectin in M. sexta (Nardi et al., 2005), calrecticulin in Galleria melonella (Choi et al., 2002), and plasmatocyte spreading peptide (PSP) in the soybean looper moth, Pseudoplusia includens Walker (Lepidoptera: Noctuidae) (Clark et al., 1997) etc. Therefore, we hypothesize that Asian corn borer plasma supplies similar molecules for the nodule formation upon the challenge of B. bassiana conidia. Future work will investigate the molecular mechanism for $O$. furncalis nodulation and clarify the exact function of plasma in nodule formation.

ACKNOWLEDGEMENTS. We thank K. He from the Institute of Plant Protection, Chinese Academy of Agricultural Sciences for kindly providing $O$. furnacalis eggs and H.D. Loxdale for his helpful editorial comments on the manuscript. This work was supported by the 973 Program (2013CB114102 and 2013CB127603), National Natural Science Foundation of China (31172090).

\section{REFERENCES}

Afidachao M.M., Musters C.J.M. \& De Snoo G.R. 2013: Asian corn borer (ACB) and non-ACB pests in GM corn (Zea mays L.) in the Philippines. - Pest Manag. Sci. 69: 792-801.

Alves e Silva T.L., Vasconcellos L.R.C., Lopes A.H. \& SoutoPAdRON T. 2013: The immune response of hemocytes of the 
insect Oncopeltus fasciatus against the flagellate Phytomonas serpens. - PloS ONE 8: e72076, 9 pp.

Bergin D., Brennan M. \& Kavanagh K. 2003: Fluctuations in haemocyte density and microbial load may be used as indicators of fungal pathogenicity in larvae of Galleria mellonella. — Microbes Infect. 5: 1389-1395.

Borges A.R., Santos P.N., Furtado A.F. \& Figueiredo B.Q. 2008: Phagocytosis of latex beads and bacteria by hemocytes of the triatomine bug Rhodnius prolixus (Hemiptera: Reduvidae). Micron 39: 486-494.

Brivio M.F., Mastore M. \& NAPPI A.J. 2010: A pathogenic parasite interferes with phagocytosis of insect immunocompetent cells. - Dev. Comp. Immunol. 34: 991-998.

Browne N., Heelan M. \& Kavanagh K. 2013: An analysis of the structural and functional similarities of insect hemocytes and mammalian phagocytes. - Virulence 4: 597-603.

Castillo J.C., Reynolds S.E. \& Eleftherianos I. 2011: Insect immune responses to nematode parasites. - Trends Parasitol. 27: $537-547$.

Cerenius L. \& Soderhall K. 2004: The prophenoloxidase-activating system in invertebrates. - Immunol. Rev. 198: 116-126.

Cherry S. \& Silverman N. 2006: Host-pathogen interactions in Drosophila: new tricks from an old friend. - Nat. Immunol. 7: 911-917.

Choi J.Y., Whitten M., Cho M.Y., Lee K.Y., Kim M.S., Ratcliffe N.A. \& LEE B.L. 2002: Calreticulin enriched as an early-stage encapsulation protein in wax moth Galleria mellonella larvae. - Dev. Comp. Immunol. 26: 335-343.

Chouvenc T., Su N. \& Robert A. 2009: Cellular encapsulation in the eastern subterranean termite, Reticulitermes flavipes (Isoptera), against infection by the entomopathogenic fungus Metarhizium anisopliae. — J. Invertebr. Pathol. 101: 234-241.

Clark K.D., Pech L.L. \& Strand M.R. 1997: Isolation and identification of a plasmatocyte-spreading peptide from the hemolymph of the lepidopteran insect Pseudoplusia includens. - J. Biol. Chem. 272: 23440-23447.

Eleftherianos I., Ffrench-Constant R.H., Clarke D.J., Dowling A.J. \& ReYnolds S.E. 2010: Dissecting the immune response to the entomopathogen Photorhabdus. - Trends Microbiol. 18: $552-560$.

Elrod-Erickson M., Mishra S. \& Schneider D. 2000: Interactions between the cellular and humoral immune responses in Drosophila. - Curr. Biol. 10: 781-784.

Ferrandon D., Imler J.L., Hetru C. \& Hoffmann J.A. 2007: The Drosophila systemic immune response: sensing and signaling during bacterial and fungal infections. - Nat. Rev. Immunol. 7: 862-874

Franc N.C., Heitzler P., Ezekowitz R. \& White K. 1999: Requirement for croquemort in phagocytosis of apoptotic cells in Drosophila. - Science 284: 1991-1994.

Franssens V., Smagghe G., Simonet G., Claeys I., Breugelmans B., Loof A. \& VAnden BroecK J. 2006: 20-hydroxyecdysone and juvenile hormone regulate the laminarin-induced nodulation reaction in larvae of the flesh fly, Neobellieria bullata. Dev. Comp. Immunol. 30: 735-740.

Hernandez S., Lanz H., Rodriguez M.H., Torres J.A., MartinezPalomo A. \& Tsutsumi V. 1999: Morphological and cytochemical characterization of female Anopheles albimanus (Diptera: Culicidae) hemocytes. - J. Med. Entomol. 36: 426-434.

Hillyer J.F. \& Strand M.R. 2014: Mosquito hemocyte-mediated immune responses. - Curr. Opin. Insect Sci. 3: 14-21.

HiLlyer J.F. 2016: Insect immunology and hematopoiesis. Dev. Comp. Immunol. 58: 102-118.

Honti V., Csordas G., Kurucz E., Markus R. \& Ando I. 2014: The cell-mediated immunity of Drosophila melanogaster: hemocyte lineages, immune compartments, microanatomy and regulation. - Dev. Comp. Immunol. 42: 47-56.

Hu J., Zhao H., Yu X., Liu J., Wang P., Chen J., Xu Q. \& Zhang W. 2010: Integrin betal subunit from Ostrinia furnacalis hemocytes: molecular characterization, expression, and effects on the spreading of plasmatocytes. - J. Insect Physiol. 56: $1846-1856$.

Hu J., Zhu X. \& Fu W. 2003: Passive evasion of encapsulation in Macrocentrus cingulum Brischke (Hymenoptera: Braconidae), a polyembryonic parasitoid of Ostrinia furnacalis Guenée (Lepidoptera: Pyralidae). — J. Insect Physiol. 49: 367-375.

Jiang H., VilcinsKas A. \& Kanost M.R. 2010: Immunity in lepidopteran insects. - Adv. Exp. Med. Biol. 708: 181-204.

Jiravanichpaisal P., Lee B.L. \& Soderhall K. 2006: Cell-mediated immunity in arthropods: hematopoiesis, coagulation, melanization and opsonization. - Immunobiology 211: 213-236.

Kanost M.R., JIANG H. \& YU X.Q. 2004: Innate immune responses of a lepidopteran insect, Manduca sexta. - Immunol. Rev. 198: $97-105$.

LACKIE A.M. 1983: Effect of substratum wettability and charge on adhesion in vitro and encapsulation in vivo by insect haemocytes. - J. Cell Sci. 63: 181-190.

Laughton A.M., Garcia J.R. \& Gerardo N.M. 2016: Conditiondependent alteration of cellular immunity by secondary symbionts in the pea aphid, Acyrthosiphon pisum. - J. Insect Physiol. 86: 17-24.

Lavine M.D. \& Strand M.R. 2002: Insect hemocytes and their role in immunity. - Insect Biochem. Mol. Biol. 32: 1295-1309.

Lemaitre B. \& Hoffmann J. 2007: The host defense of Drosophila melanogaster. - Annu. Rev. Immunol. 25: 697-743.

Ling E., Shirai K., Kanekatsu R. \& Kiguchi K. 2005: Hemocyte differentiation in the hematopoietic organs of the silkworm, Bombyx mori: prohemocytes have the function of phagocytosis. - Cell Tissue Res. 320: 535-543

Liu Y., Shen D., Zhou F., Wang G. \& An C. 2014: Identification of immunity-related genes in Ostrinia furnacalis against entomopathogenic fungi by RNA-seq analysis. - PloS ONE 9: e86436, 24 pp.

Mastore M., Arizza V., Manachini B. \& Brivio M.F. 2015a: Modulation of immune responses of Rhynchophorus ferrugineus (Insecta: Coleoptera) induced by the entomopathogenic nematode Steinernema carpocapsae (Nematoda: Rhabditida). - Insect Sci. 22: 748-760.

Mastore M., Binda R.S., Giovannardi S., Scari G. \& Brivio M.F. 2015b: Inducible factors with antimicrobial activity after immune challenge in the haemolymph of Red Palm Weevil (Insecta). - Innate Immun. 21: 392-405.

Moita L.F., VRiend G., Mahairaki V., Louis C. \& Kafatos F.C. 2006: Integrins of Anopheles gambiae and a putative role of a new beta integrin, BINT2, in phagocytosis of Escherichia coli. — Insect Biochem. Mol. Biol. 36: 282-290.

Nappi A.J. \& OtTaViani E. 2000: Cytotoxicity and cytotoxic molecules in invertebrates. - Bioessays 22: 469-480.

NaqQASH M.N., GoKce A., BAKHSh A. \& SAlim M. 2016: Insecticide resistance and its molecular basis in urban insect pests. - Parasitol. Res. 115: 1363-1373.

Nardi J.B., Zhuang S.F., Pilas B., Bee C.M. \& Kanost M.R. 2005: Clustering of adhesion receptors following exposure of insect blood cells to foreign surfaces. - J. Insect Physiol. 51: 555-564.

Pearson A., Lux A. \& Krieger M. 1995: Expression cloning of dsr-ci, a class-c macrophage-specific scavenger receptor from Drosophila melanogaster. - Proc. Natl. Acad. Sci. U.S.A. 92: 4056-4060. 
Ramet M., Manfruelli P., Pearson A., Mathey-Prevot B. \& EzEKowITZ R. 2002: Functional genomic analysis of phagocytosis and identification of a Drosophila receptor for Escherichia coli. - Nature 416: 644-648.

RATCLIFFE N.A. \& GAGEN S.J. 1977: Studies on in vivo cellular reactions of insect ultrastructural analysis of nodule formation in Galleria mellonella. — Tissue Cell 9: 73-85.

Sanghamitra S., Mohanty A.K., Parthasarathi S., Mishra S.K., Behera P.K., Goutam P. \& Dondorp A.M. 2014: Comparing Leishman and Giemsa staining for the assessment of peripheral blood smear preparations in a malaria endemic region in India. - Malaria J. 13: 30-2014.

Scherfer C., Qazi M.R., Takahashi K., Ueda R., Dushay M.S., Theopold U. \& Lemaitre B. 2006: The toll immune regulated Drosophila protein Fondue is involved in hemolymph clotting and puparium formation. - Dev. Biol. 295: 156-163.

Shu M., Mang D., Fu G.S., Tanaka S., Endo H., Kikuta S. \& SATO R. 2016: Mechanisms of nodule-specific melanization in the hemocoel of the silkworm, Bombyx mori. - Insect Biochem. Mol. Biol. 70: 10-23.

Silva J.B., Albuquerque C., Araujo E.C., Peixoto C.A. \& Hurd H. 2000: Immune defense mechanisms of Culex quinquefasciatus (Diptera: Culicidae) against Candida albicans infection. — J. Invertebr. Pathol. 76: 257-262.

Strand M.R. \& Pech L.L. 1995: Immunological basis for compatibility in parasitoid-host relationships. - Annu. Rev. Entomol. 40: 31-56.

Taskin B.G., Dogaroglu T., Kilic S., Dogac E. \& Taskin V. 2016: Seasonal dynamics of insecticide resistance, multiple resistance, and morphometric variation in field populations of Culex pipiens. - Pestic Biochem. Physiol. 129: 14-27.

Theopold U., Schmidt O., Soderhall K. \& Dushay M.S. 2004: Coagulation in arthropods: defence, wound closure and healing. - Trends Immunol. 25: 289-294.
Vogelweith F., Moret Y., Monceau K., Thiery D. \& Moreau J. 2016: The relative abundance of hemocyte types in a polyphagous moth larva depends on diet. - J. Insect Physiol. 88: 33-39.

Wagner B.L. \& Lewis L.C. 2000: Colonization of corn, Zea mays, by the entomopathogenic fungus Beauveria bassiana. Appl. Environ. Microb. 66: 3468-3473.

Wu G., YI Y., Sun J., Li M. \& QIU L. 2015: No evidence for priming response in Galleria mellonella larvae exposed to toxin protein PirA2B2 from Photorhabdus luminescens TT01: An association with the inhibition of the host cellular immunity. Vaccine 33: 6307-6313.

Xu Q., Yu X., Jia L., Zhao H., Peng W., Hu S., Chen J., Zhang W. \& JIAN H. 2012: Ostrinia furnacalis integrin $\beta 1$ may be involved in polymerization of actin to modulate spreading and encapsulation of plasmatocytes. - Dev. Comp. Immunol. 37: 438-445.

Yoкоо S., Gotz P. \& Tojo S. 1995: Phagocytic activities of hemocytes separated by 2 simple methods from larvae of 2 lepidopteran species, Agrotis segetum and Galleria mellonella. - Appl. Entomol. Zool. 30: 343-350.

Zhuang S., Kelo L., Nardi J.B. \& Kanost M.R. 2008: Multiple alpha subunits of integrin are involved in cell-mediated responses of the Manduca immune system. - Dev. Comp. Immunol. 32: 365-379.

Zibaee A., Bandani A.R., Talaei-Hassamlouei R. \& Malagoli D. 2011: Cellular immune reactions of the sunn pest, Eurygaster integriceps, to the entomopathogenic fungus, Beauveria bassiana and its secondary metabolites. - J. Insect Sci. 138: 1-16.

Received March 11, 2016; revised and accepted May 2, 2016 Published online July 8, 2016 